\title{
Effect of cosyntropin during adrenal venous sampling on subtype of primary aldosteronism: analysis of surgical outcome
}

\author{
Hiroki Kobayashi,2,3, Yoshihiro Nakamura', Masanori Abe1, Isao Kurihara4, Hiroshi Itoh4, Takamasa Ichijo5, \\ Yoshiyu Takeda ${ }^{6}$, Takashi Yoneda6, Takuyuki Katabami , Mika Tsuiki ${ }^{8}$, Norio Wada9 $^{9}$ Yoshihiro Ogawa ${ }^{10}$, \\ Ryuichi Sakamoto ${ }^{10}$, Junji Kawashima11, Masakatsu Sone ${ }^{12}$, Nobuya Inagaki'12, Takanobu Yoshimoto ${ }^{13,14}$, \\ Tetsuya Yamada ${ }^{14}$, Ryuji Okamoto ${ }^{15}$, Yuichi Matsuda ${ }^{16}$, Megumi Fujita $^{17}$, Minemori Watanabe ${ }^{18}$, \\ Kouichi Tamura ${ }^{19}$, Akiyo Tanabe ${ }^{20}$ and Mitsuhide Naruse ${ }^{8}$ on behalf of JPAS/JRAS Study Group
}

${ }^{1}$ Division of Nephrology, Hypertension, and Endocrinology, Nihon University School of Medicine, Tokyo, Japan, ${ }^{2}$ Section on Genetics and Epidemiology, Research Division, Joslin Diabetes Center, Boston, Massachusetts, USA, ${ }^{3}$ Department of Medicine, Harvard Medical School, Boston, Massachusetts, USA, ${ }^{4}$ Department of Endocrinology, Metabolism, and Nephrology, Keio University School of Medicine, Tokyo, Japan, ${ }^{5}$ Department of Diabetes and Endocrinology, Saiseikai Yokohamashi Tobu Hospital, Yokohama, Japan, ${ }^{6}$ Department of Internal Medicine, Graduate School of Medical Science, Kanazawa University, Kanazawa, Japan, ${ }^{7}$ Division of Metabolism and Endocrinology, Department of Internal Medicine, St. Marianna University School of Medicine, Yokohama City Seibu Hospital, Yokohama, Japan, ${ }^{8}$ Department of Endocrinology and Metabolism, National Hospital Organization Kyoto Medical Center, Kyoto, Japan, ${ }^{9}$ Department of Diabetes and Endocrinology, Sapporo City General Hospital, Sapporo, Japan, ${ }^{10}$ Department of Medicine and Bioregulatory Science, Kyushu University, Fukuoka, Japan, ${ }^{11}$ Department of Metabolic Medicine, Faculty of Life Science, Kumamoto University, Kumamoto, Japan, ${ }^{2}$ Department of Diabetes, Endocrinology and Nutrition, Kyoto University, Kyoto, Japan, ${ }^{13}$ Department of Diabetes and Endocrinology, Tokyo Metropolitan Hiroo Hospital, Tokyo, Japan, ${ }^{14}$ Department of Molecular Endocrinology and Metabolism, Tokyo Medical and Dental University, Tokyo, Japan, ${ }^{15}$ Department of Cardiology and Nephrology, Mie University Graduate School of Medicine, Tsu, Japan, ${ }^{16}$ Department of Cardiology, Sanda City Hospital, Sanda, Japan, ${ }^{17}$ Division of Nephrology and Endocrinology, University of Tokyo, Tokyo, Japan, ${ }^{18}$ Department of Endocrinology and Diabetes, Okazaki City Hospital, Okazaki, Japan, ${ }^{19}$ Department of Medical Science and Cardiorenal Medicine, Yokohama City University Graduate School of Medicine, Yokohama, Japan, and ${ }^{20}$ Division of Endocrinology, National Center for Global Health and Medicine, Tokyo, Japan

Correspondence should be addressed to H Kobayashi or M Abe Email kobayashi.hiroki@nihon-u. ac.jp or abe.masanori@ nihon-u.ac.jp

\section{Abstract}

Objectives: We investigated the clinical significance of ACTH stimulation during adrenal venous sampling (AVS) by surgical outcome of primary aldosteronism (PA).

Design: Multicenter retrospective study by Japan PA study.

Method: We allocated 314 patients with both basal and ACTH-stimulated AVS data who underwent adrenalectomy to three groups: basal lateralization index (LI) $\geq 2$ with $\mathrm{ACTH}$-stimulated $\mathrm{LI} \geq 4$ on the ipsilateral side (Unilateral (U) to $\mathrm{U}$ group, $n=245$ ); basal $\mathrm{LI}<2$ with $\mathrm{ACTH}$-stimulated $\mathrm{LI} \geq 4$ (Bilateral (B) to $\mathrm{U}$ group, $n=15$ ); and basal $\mathrm{LI} \geq 2$ with ACTH-stimulated $\mathrm{LI}<4$ ( $U$ to B group, $n=54$ ). We compared surgical outcomes among the groups using the Primary Aldosteronism Surgical Outcome (PASO) criteria.

Results: Compared with $U$ to $U$ group, $U$ to $B$ group had poor clinical and biochemical outcomes and low rates of adrenal adenoma as pathological findings $(P=0.044,0.006$, and 0.048 , respectively), although there were no significant differences between $U$ to $U$ and $B$ to $U$ groups. All patients in $U$ to B group with clinical and biochemical benefits, however, had adrenal adenoma as pathological findings and could be well differentiated from those with poor surgical outcomes via basal LI (>8.3), but not ACTH-stimulated LI. These results were similar even when we defined each group based on a cut-off value of 4 for basal LI.

Conclusions: Although PA patients in $U$ to B group had worse surgical outcomes than did those in $U$ to $U$ group, basal $\mathrm{LI}$ could discriminate among patients with better surgical outcomes in $U$ to B group. 


\section{Introduction}

Primary aldosteronism (PA), a major cause of secondary hypertension, has an estimated prevalence of approximately $10 \%$ among patients with hypertension $(1,2,3,4)$. Compared to patients with essential hypertension, those with PA are more frequently affected by cardiovascular diseases due to the hyperaldosteronism effect $(5,6,7)$. Thus, early diagnosis and treatment of excessive aldosterone production are mandatory to prevent its complications. All current guidelines recommend adrenal venous sampling (AVS) for PA subtyping (1, 8), that is, for distinguishing between the lateralized form, mainly aldosterone-producing adenoma (APA) treated via adrenalectomy and bilateral adrenocortical hyperplasia treated with medication. However, many of its technical aspects and interpretation remain controversial among various AVS specialist centers $(9,10,11,12)$. Whether we should use adrenocorticotropic hormone (ACTH) stimulation during AVS has been widely disputed without any conclusions (12). Exogenous synthetic ACTH is administered during AVS in some centers to minimize stress-induced fluctuations in aldosterone secretion, maximize the gradient in cortisol from the adrenal vein to the inferior vena cava, maximize aldosterone secretion from APAs, and improve the success rate of bilaterally selective catheterization. While some studies showed that ACTH stimulation during AVS leads to correct lateralization, others showed the opposite $(10,13,14)$. Therefore, recent AVS studies showed that about $40 \%$ of centers worldwide use ACTH stimulation during AVS $(9,14)$. Accordingly, this study evaluated the clinical implications of ACTH stimulation during AVS in terms of outcomes after adrenalectomy, using a large multi-center dataset. Furthermore, we assessed predictors of surgical outcomes among patients with divergent lateralization results of AVS with and without ACTH stimulation.

\section{Methods}

\section{Setting and subjects}

We performed a retrospective observational cohort study of patients enrolled in the Japan Rare/Intractable Adrenal Disease Study (JRAS) data, a nationwide registry of patients from 41 referral centers. The study protocol was approved by the Ethics Committee of the National Hospital Organization Kyoto Medical Center (Kyoto, Japan) (the lead center) and the institutional ethics committees of the participating centers. The study was registered with the
University Hospital Medical Information Network (UMIN ID: 18756). The JRAS included patients with PA, aged 20-90 years, who underwent AVS. We used the JRAS dataset that was available in May 2019, which included patients registered between January 2006 and December 2018. Detailed information about PA diagnosis is shown in the Supplementary methods (see section on supplementary materials given at the end of this article). Due to the retrospective nature of the present study, the indications for adrenalectomy per case were determined by physicians at each center based on the AVS results, as well as on other clinical characteristics, such as CT imaging and PA severity. We included patients with AVS data before and after ACTH stimulation and those who underwent adrenalectomy, with 6-12-month follow-up data to evaluate the surgical outcome. Patients with incomplete AVS data, failure of cannulation, or autonomous cortisol secretion (defined as a cortisol level of $\geq 3.0 \mu \mathrm{g} / \mathrm{dL}$ after a $1.0 \mathrm{mg}$ dexamethasone suppression test) were excluded from the analysis (15).

\section{Outcome assessment}

The Primary Aldosteronism Surgical Outcome (PASO) investigators developed an international consensus for the assessment of outcomes after unilateral adrenalectomy for PA (16). In this consensus, clinical and biochemical outcomes were classified as complete, partial, or absent, based on the surgical results. Clinical outcome is defined by the change of blood pressure and number of antihypertensive drugs, and biochemical outcome is defined by the change of plasma aldosterone concentration (PAC) and aldosterone/renin ratio (ARR). Detailed information on the outcome criteria has been previously described (16). Outcome assessment was carried out at 6-12 months after surgery.

\section{Adrenal venous sampling}

Detailed information about the AVS procedure has been described previously $(17,18)$. Briefly, adrenal blood samples were obtained via sequential cannulation by the percutaneous femoral vein approach. ACTH was administered by bolus injection, bolus injection followed by continuous infusion, or continuous infusion only, throughout the procedure. Adrenal vein cannulation was defined as successful if the selectivity index was $\geq 2$ at basal levels and $\geq 5$ after ACTH stimulation (9). Unilateral hyperaldosteronism on AVS was defined as a lateralization index (LI) of $\geq 2$ at the baseline and $\geq 4$ after ACTH use, which are the most commonly used cut-off values $(9,19)$. 
The selectivity index was defined as the ratio of the cortisol level in the adrenal vein to that in the inferior vena cava. The LI was calculated by dividing the aldosterone/cortisol ratio on the dominant side by that on the non-dominant side. The contralateral aldosterone suppression ratio (CR) was calculated by dividing the aldosterone-to-cortisol ratio in the nondominant adrenal vein by that in the inferior vena cava. To compare between discrepant LI values assessed before and after ACTH stimulation, we allocated the patients into three groups: those with basal LI $\geq 2$ and ACTH stimulated LI $\geq 4$ on the ipsilateral side (Unilateral (U) to U group); basal LI $<2$ and ACTH stimulated LI $\geq 4$ (Bilateral (B) to U group); and basal $\mathrm{LI} \geq 2$ and $\mathrm{ACTH}$ stimulated $\mathrm{LI}<4$ ( $\mathrm{U}$ to $\mathrm{B}$ group). In the sensitivity analysis, we repeated the main analysis by using groups based on the basal LI cut-off value of $4(20,21)$. Next, we also analyzed only for patients whose ACTH stimulation were administered by bolus injection.

\section{Statistical analysis}

Continuous variables are expressed as the mean \pm S.D. or median (interquartile range). Continuous variables were compared using the Mann-Whitney $U$ test. Fisher's exact test was used to compare the categorical variables. Statistical significance was set at $P<0.05$. Since surgery is typically indicated for patients in the $\mathrm{U}$ to $\mathrm{U}$ group, we compared patients in $\mathrm{U}$ to $\mathrm{U}$ group with those in the other two groups. To compare the surgical outcomes, a Bonferroni correction was calculated based on the number of comparisons between groups. Next, to assess good surgical outcomes in the $\mathrm{U}$ to $\mathrm{B}$ group, we ran multivariable logistic regression analysis with backward selection. In the model, we included variables that were significantly different between patients with good and bad surgical outcomes in the univariate analysis. Receiver operating characteristic curve analyses were then used to assess the diagnostic ability of basal LI in the $\mathrm{U}$ to $\mathrm{B}$ group. All statistical analyses were performed using SPSS, version 22 (IBM Corp.).

\section{Results}

\section{Study population}

Of the 3689 patients who had information in the JRAS dataset, we excluded 1722 with missing or incomplete information regarding basal or ACTH stimulated AVS data and 113 with autonomous cortisol secretion. Figure 1 and Supplementary Figure 1 show the proportion of patients in each group defined by the pre- and post-ACTH-stimulated

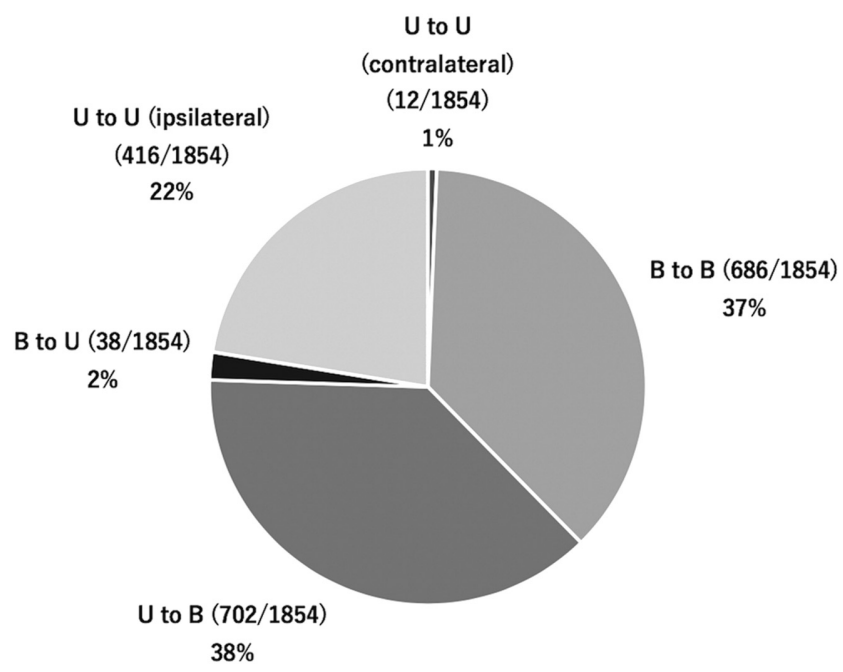

\section{Figure 1}

Prevalence of each group defined by basal and ACTH stimulated laterality index on adrenal venous sampling. Unilateral hyperaldosteronism on AVS was defined as a lateralization index of $\geq 2$ at the baseline and $\geq 4$ after ACTH stimulation. $U$ to $\mathrm{U}$, unilateral to unilateral; $\mathrm{B}$ to $\mathrm{U}$, bilateral to unilateral; $\mathrm{U}$ to $\mathrm{B}$, unilateral to bilateral; $\mathrm{B}$ to $\mathrm{B}$, bilateral to bilateral.

$\mathrm{LI}$ ( $\mathrm{U}$ to $\mathrm{U}, \mathrm{B}$ to $\mathrm{U}, \mathrm{B}$ to $\mathrm{U}$, and $\mathrm{B}$ to $\mathrm{B}$ groups) among the remaining 1854 patients. The $U$ to $B$ group had the highest proportion of patients (38\%; 702/1854). Conversely, only $2 \%$ of patients were in B to U group (38/1854). Among the 1854 patients, 422 underwent adrenalectomy and 83 were excluded due to the missing 6-12-month follow-up data after adrenalectomy. Among the total of 339 patients, 19 had bilateral PA in both basal and ACTH-stimulated AVS and six had opposite-side unilateral PA between basal and ACTH-stimulated AVS. Thus, a total of 314 were included among the three groups in the present analysis. In these patients, ACTH was administered by either bolus injection ( $n=203)$, bolus injection followed by continuous infusion $(n=88)$, or continuous infusion only $(n=23)$, throughout the procedure. The baseline clinical characteristics of patients in each group are shown in Table 1. Compared with patients in the $U$ to $U$ group, patients in other groups had significantly lower PAC, ARR, basal LI, and ACTH stimulated LI, and higher basal CR and ACTH stimulated CR.

\section{Comparison of surgical outcomes among the groups}

Next, we compared the surgical outcomes (clinical and biochemical benefit) and adrenal adenoma prevalence, 
Table 1 Clinical characteristics of primary aldosteronism in each group. Data are presented as the mean ( \pm S.D.), median (interquartile range), or percentage.

\begin{tabular}{l} 
Characteristic \\
\hline Age (years) \\
Sex (male) \\
BMI (kg/m²) \\
SBP (mmHg) \\
DBP (mmHg) \\
Oral potassium supplementation (\%) \\
Serum potassium level (mmol/L) \\
eGFR (mL/min/1.73 m $^{2}$ ) \\
PAC (pg/mL) \\
PRA (ng/mL/h) \\
ARR \\
LI (pre-ACTH stimulation) \\
CR (pre-ACTH stimulation) \\
LI (post-ACTH stimulation) \\
CR (post-ACTH stimulation) \\
Prevalence of adrenal nodule \\
$\quad$ on CT imaging (\%)
\end{tabular}

\begin{tabular}{c}
$\begin{array}{c}\text { Unilateral to } \\
\text { Unilateral }(n=245)\end{array}$ \\
\hline $50( \pm 12)$ \\
$53 \%(130)$ \\
$24.5( \pm 4.2)$ \\
$141( \pm 18)$ \\
$87( \pm 12)$ \\
$52 \%(128)$ \\
$3.3( \pm 0.56)$ \\
$80.4( \pm 21.1)$ \\
$322(220-447)$ \\
$0.30(0.10-0.40)$ \\
$1270(635-2348)$ \\
$19.8(8.7-48.1)$ \\
$0.33(0.19-0.59)$ \\
$13.5(8.1-28.2)$ \\
$0.29(0.17-0.51)$ \\
$79 \%(194)$
\end{tabular}

\begin{tabular}{c} 
Bilateral to \\
Unilateral $(n=15)$ \\
\hline $54( \pm 9)$ \\
$60 \%(9)$ \\
$25.0( \pm 4.7)$ \\
$143( \pm 13)$ \\
$85( \pm 11)$ \\
$33 \%(5)$ \\
$3.6( \pm 0.45)$ \\
$77.7( \pm 21.1)$ \\
$181(139-302)$ \\
$0.30(0.20-0.40)$ \\
$645(550-1290)$ \\
$1.4(1.3-1.8)$ \\
$1.2(0.37-1.7)$ \\
$5.7(4.8-11.2)$ \\
$0.72(0.27-0.93)$ \\
$60 \%(9)$
\end{tabular}

\begin{tabular}{c}
$\begin{array}{c}\text { Unilateral to } \\
\text { Bilateral }(n=54)\end{array}$ \\
\hline $51( \pm 11)$ \\
$46 \%(25)$ \\
$24.7( \pm 3.1)$ \\
$135( \pm 20)$ \\
$83( \pm 15)$ \\
$35 \%(19)$ \\
$3.5( \pm 0.58)$ \\
$84.0( \pm 19.4)$ \\
$243(156-300)$ \\
$0.30(0.20-0.43)$ \\
$686(481-1250)$ \\
$6.0(3.0-13.8)$ \\
$0.60(0.37-1.7)$ \\
$2.7(2.2-3.2)$ \\
$0.88(0.38-1.6)$ \\
$72 \%(39)$
\end{tabular}

\begin{tabular}{|c|c|}
\hline $\begin{array}{c}\text { P value (U to U } \\
\text { vs B to U) }\end{array}$ & $\begin{array}{c}\text { P value ( } U \text { to } U \\
\text { vs } \cup \text { to } B \text { ) }\end{array}$ \\
\hline 0.25 & 0.87 \\
\hline 0.79 & 0.37 \\
\hline 0.73 & 0.47 \\
\hline 0.46 & 0.037 \\
\hline 0.60 & 0.071 \\
\hline 0.11 & 0.029 \\
\hline 0.061 & 0.013 \\
\hline 0.56 & 0.24 \\
\hline 0.003 & $<0.001$ \\
\hline 0.43 & 0.20 \\
\hline 0.025 & $<0.001$ \\
\hline$<0.001$ & $<0.001$ \\
\hline$<0.001$ & $<0.001$ \\
\hline 0.002 & $<0.001$ \\
\hline 0.002 & $<0.001$ \\
\hline 0.11 & 0.28 \\
\hline
\end{tabular}

ARR, aldosterone/renin ratio; $\mathrm{BMI}$, body mass index; $\mathrm{CR}$, contralateral ratio; $\mathrm{CT}$, computed tomography; DBP, diastolic blood pressure; eGFR, estimated glomerular filtration rate; LI, lateralization index; PAC, plasma aldosterone concentration; PRA, plasma renin activity; SBP, systolic blood pressure.

as a pathological finding, between the $\mathrm{U}$ to $\mathrm{U}$ group and the other groups. As shown in Fig. 2, both clinical and biochemical benefit rates were nominally and significantly lower in the $\mathrm{U}$ to $\mathrm{B}$ group than in the $\mathrm{U}$ to $\mathrm{U}$ group $(P=0.044$ and 0.006 , respectively). In addition, the rate of adrenal adenoma, as a pathological finding, was nominally and significantly lower in the $U$ to $B$ group than in the $U$ to $U$ group $(P=0.048)$. The difference of biochemical outcome reached the significance threshold after Bonferroni correction (adjusted $P=0.012$ ). In the sensitivity analysis, the significance of difference in biochemical success between groups was retained even when we defined each group using a basal LI cut-off value of 4 (adjusted $P=0.048$ ) and when we analyzed only for patients whose ACTH stimulation were administered by bolus injection (adjusted $P=0.004)(n=203)$ (Supplementary Figs 2 and 3).

\section{Prediction of better surgical outcome in the $\mathrm{U}$ to $\mathrm{B}$ patient group}

From these results, we hypothesized that the $U$ to $B$ group comprised patients with both surgically curable unilateral APA and non-curable bilateral hyperaldosteronism. Thus, we assessed the surgical outcome predictors among 54 patients in the $U$ to $B$ group. Initially, to evaluate the potential for selection bias in assessing only patients who underwent adrenalectomy, we compared the clinical characteristics between patients who underwent adrenalectomy $(n=54)$ and those who did not $(n=632)$ in the $\mathrm{U}$ to $\mathrm{B}$ group. As shown in Supplementary Table 1, patients who underwent adrenalectomy showed significantly higher PAC, ARR, and LI (pre- and post-
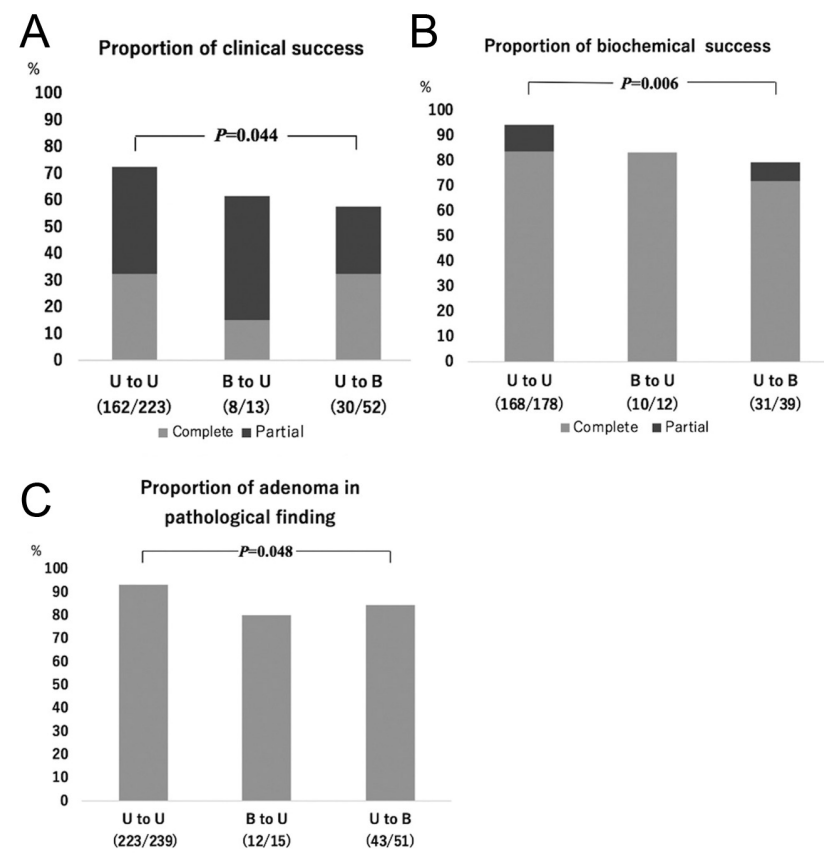

\section{Figure 2}

Clinical outcomes (A), biochemical outcomes (B), and pathological findings (C) after adrenalectomy for each group. $U$ to $U$, unilateral to unilateral; $B$ to $U$, bilateral to unilateral; $U$ to $\mathrm{B}$, unilateral to bilateral; $\mathrm{B}$ to $\mathrm{B}$, bilateral to bilateral. 
ACTH stimulation) $(P<0.001,<0.001,0.020$, and $<0.001$, respectively) and lower systolic blood pressure, diastolic blood pressure, serum potassium level, and CR (pre-and post-ACTH stimulation) $(P=0.012,0.025,<0.001,0.0018$, and $<0.001$, respectively) than did those who did not undergo adrenalectomy. In addition, a higher proportion of patients who underwent adrenalectomy received potassium supplementation and showed adrenal nodule on CT scan (both $P<0.001$ ), indicating that patients who underwent adrenalectomy had more florid cases of APA.

Next, we compared the clinical characteristics between the $U$ to $B$ group with and without clinical or biochemical benefit after adrenalectomy. Importantly, basal LI and CR, but not ACTH stimulated LI and CR, differed significantly between patients with and without clinical benefit (both $P<0.001$ ) (Supplementary Table 2). Furthermore, patients with and without biochemical benefit showed significant differences in basal CR $(P=0.011)$ (Supplementary Table 3). These results were similar to those obtained based on the groups defined according to the basal LI cut-off value of 4 and those obtained only in patients whose ACTH stimulation were administered by bolus injection (Supplementary Tables 4 and 5). We assessed the predictors of both clinical and biochemical benefits after adrenalectomy. All patients (19/19) with both clinical and biochemical benefits had adrenal adenoma as a pathological finding after adrenalectomy. To ascertain whether these patients were cured of PA, we were able to evaluate the long-term follow-up data of 12 of the

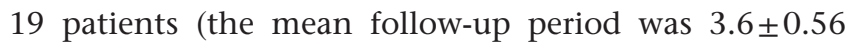
years). During the follow-up period, 11 of the 12 patients showed persistent, complete biochemical success. Only one patient had PA recurrence confirmed via a positive captopril challenge test result after 3 years of follow-up. The details of this case are shown in Supplementary Table 6. Notably, the AVS result of this case showed that the basal aldosterone concentrations in the adrenal veins on both the dominant and non-dominant sides were high (18 $600 \mathrm{pg} / \mathrm{mL}$ and $15400 \mathrm{pg} / \mathrm{mL}$, respectively). However, the basal LI was high (13.5) because the cortisol level obtained on the non-dominant side was far higher than that obtained on the dominant side $(1130 \mathrm{pg} / \mathrm{mL}$ vs 101 $\mathrm{pg} / \mathrm{mL}$ ). These data imply that we might have been able to diagnose this case as bilateral hyperaldosteronism using the absolute value of the basal aldosterone level in the adrenal veins.

As shown in Table 2, patients with both clinical and biochemical benefits had significantly higher BMI, ARR, and basal LI values, and a lower CR value than those with absent clinical or biochemical success $(P=0.014, P=0.016$, $P=0.0023$, and $P<0.001$, respectively). Multivariable logistic regression analysis with backward selection showed that BMI and basal LI were independent predictors of both clinical and biochemical benefits (odds ratio $=0.60$, $P=0.015$ and odds ratio $=1.21, P=0.015$, respectively) (Table 3). In the receiver operating characteristic curve

Table 2 Baseline characteristics of patients in the unilateral to bilateral group with and without clinical and biochemical benefits after adrenalectomy. Continuous data are shown as the mean ( \pm S.D.) and median (interquartile range).

\begin{tabular}{l}
\hline \\
\hline Age (years) \\
Sex, males/females \\
Body mass index $\left(\mathrm{kg} / \mathrm{m}^{2}\right)$ \\
Systolic blood pressure (mmHg) \\
Diastolic blood pressure (mmHg) \\
Oral potassium supplementation (\%) \\
Serum potassium (mmol/L) \\
eGFR (mL/min/1.73 m²) \\
Baseline PAC (pg/mL) \\
Baseline PRA (ng/mL/h) \\
Baseline ARR \\
Laterality index (pre-ACTH stimulation) \\
Contralateral suppression (pre-ACTH stimulation) \\
Laterality index (post-ACTH stimulation) \\
Contralateral suppression (post-ACTH stimulation) \\
Prevalence of adrenal nodule on CT imaging (\%) \\
Diameter of adrenal nodule on CT imaging (mm) \\
Prevalence of adrenal adenoma on pathology (\%) \\
LRR
\end{tabular}

\begin{tabular}{c}
$\begin{array}{c}\text { Clinical and biochemical } \\
\text { success }(n=19)\end{array}$ \\
\hline $49.6( \pm 11.9)$ \\
$7 / 12$ \\
$23.4( \pm 2.5)$ \\
$135.5( \pm 25.5)$ \\
$83.8( \pm 15.8)$ \\
$47(9)$ \\
$3.3( \pm 0.54)$ \\
$87.6( \pm 20.9)$ \\
$279(170-454)$ \\
$0.20(0.20-0.30)$ \\
$947(603-2610)$ \\
$13.5(5.1-31.5)$ \\
$0.40(0.19-0.56)$ \\
$2.8(2.3-3.6)$ \\
$0.81(0.24-1.8)$ \\
$90(17)$ \\
$15.0(11.0-17.0)$ \\
$100(19)$
\end{tabular}

\begin{tabular}{ccc}
\hline $\begin{array}{c}\text { Clinical or biochemical } \\
\text { failure }(n=25)\end{array}$ & & P value \\
\hline $53.1( \pm 10.8)$ & & 0.37 \\
$15 / 10$ & & 0.22 \\
$25.7( \pm 3.1)$ & & 0.014 \\
$131.4( \pm 13.9)$ & & 0.53 \\
$80.6( \pm 15.3)$ & & 0.42 \\
$24(6)$ & & 0.12 \\
$3.6( \pm 0.65)$ & & 0.036 \\
$83.3( \pm 18.7)$ & & 0.49 \\
$196(154-263)$ & & 0.074 \\
$0.40(0.20-0.60)$ & & 0.039 \\
$600(336-870)$ & & 0.016 \\
$3.8(2.4-6.9)$ & & 0.0023 \\
$1.4(0.75-2.5)$ & & $<0.001$ \\
$2.6(2.4-3.1)$ & & 0.47 \\
$1.0(0.60-1.6)$ & & 0.21 \\
$72(18)$ & & 0.26 \\
$12.0(10.5-14.3)$ & & 0.27 \\
$73(16)(n=22)$ & & 0.023 \\
\hline
\end{tabular}

ARR, aldosterone-renin ratio; CT, computed tomography; eGFR, estimated glomerular filtration rate; PAC, plasma aldosterone concentration; PRA, plasma renin activity. 
Table 3 Multivariable logistic regression model for the prediction of both clinical and biochemical benefits. Body mass index, serum potassium concentration, plasma renin activity, laterality index (pre-ACTH stimulation), and contralateral ratio (pre-ACTH stimulation) were included in the multivariable model.

\begin{tabular}{|c|c|c|}
\hline Characteristic & Odds ratio $(95 \% \mathrm{Cl})$ & $P$ value \\
\hline Body mass index & $0.60(0.40-0.90)$ & 0.015 \\
\hline $\begin{array}{l}\text { Laterality index } \\
\quad \text { (pre-ACTH stimulation) }\end{array}$ & $1.21(1.04-1.41)$ & 0.015 \\
\hline
\end{tabular}

Body mass index, serum potassium concentration, plasma renin activity, laterality index (pre-ACTH stimulation), and contralateral ratio (pre-ACTH stimulation) were included in the multivariable model.

analysis of basal LI, a cut-off value of 8.3 showed a specificity of $84 \%$ and sensitivity of $63 \%$, while a cut-off value of 6.3 showed a specificity of $72 \%$ and sensitivity of $74 \%$ for the prediction of both clinical and biochemical benefit in the $\mathrm{U}$ to B group (area under the curve: 0.77 , 95\% CI: 0.622-0.920) (Fig. 3). The sensitivity analysis showed similar results (Supplementary Tables 7 and 8).

\section{Differences in aldosterone and cortisol levels in the adrenal veins between the $U$ to $B$ and $U$ to $U$ groups}

To evaluate the specific differences between patients with better surgical outcomes in the $U$ to $B$ and $U$ to $U$ groups, we compared the values of PAC, plasma cortisol concentration, and aldosterone/cortisol ratios obtained on the dominant and non-dominant sides in the adrenal vein before and after ACTH stimulation (Fig. 4).

The basal PAC, plasma cortisol concentration, and aldosterone/cortisol ratios in the adrenal veins on both sides among patients with better surgical outcome in the $U$ to B group were not significantly different from those in the $U$ to $U$ group. On the other hand, there was a significant difference in the ACTH stimulated PAC on the dominant side (33 608 \pm 36652 vs $104464 \pm 112107$ pg/ $\mathrm{mL}, P<0.001$, respectively). These results implied that the important difference between patients in both groups was the aldosterone response by ACTH stimulation.

\section{Discussion}

Despite the high PA prevalence, the only reliable method for selecting patients with PA who could be treated via adrenalectomy is AVS, which is undertaken in different ways in different institutions. In particular, it has

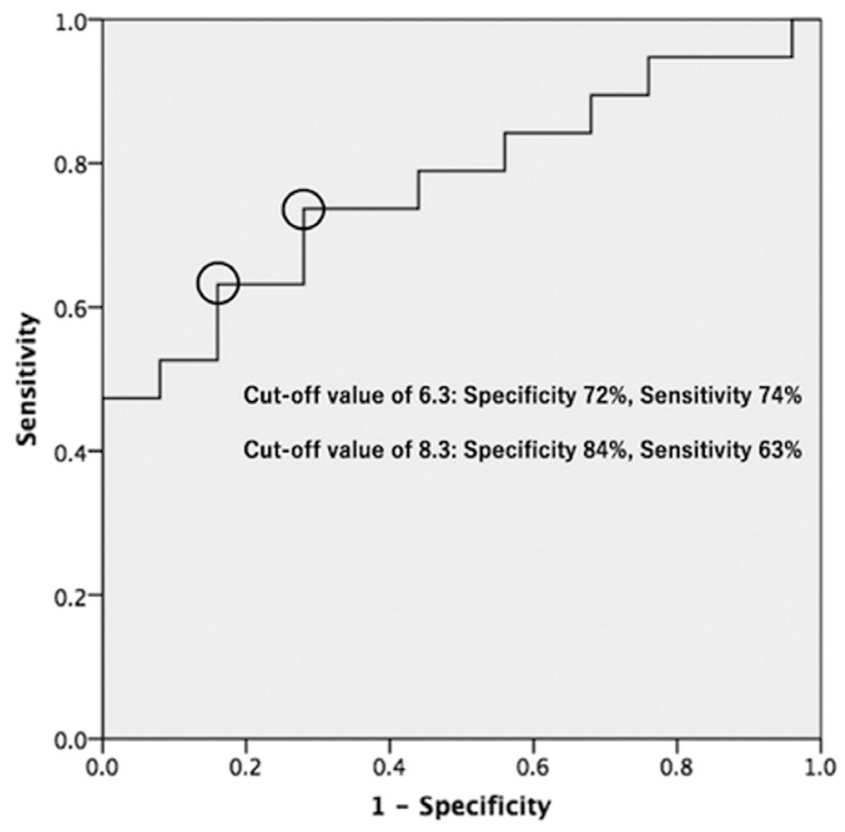

Figure 3

Receiver-operating characteristic curve of basal lateralization index for the prediction of better surgical outcome in the $U$ to B group.

been widely discussed whether we should use ACTH stimulation during AVS; however, no consensus has been reached. In our previous study, we demonstrated that ACTH stimulation increases the success rate of bilateral selective catheterization (18). However, we could not show whether or not ACTH stimulation affected the outcome of adrenalectomy due to the inadequate sample size.

In this study, using the largest database of PA patients who underwent AVS, we demonstrated for the first time that PA in the U to B group had worse clinical and biochemical outcomes and a lower rate of adrenal adenoma, as a pathological finding, after adrenalectomy than did those in the $U$ to $U$ group. In the $U$ to B group, all patients with both clinical and biochemical benefits after adrenalectomy had adrenal adenoma as a pathological finding, and strikingly, these patients with better surgical outcomes could be differentiated from those with worse surgical outcomes using basal LI, but not ACTH-stimulated LI. These results were almost concordant even when we defined the cutoff value of 4 for basal LI and when we analyzed only for patients whose ACTH stimulation was administered by bolus injection.

Some previous studies that compared basal and ACTH-stimulated AVS results yielded contrary findings, including decreased, unchanged, or increased ACTHstimulated LI from the basal value $(14,22,23)$. Another 
A

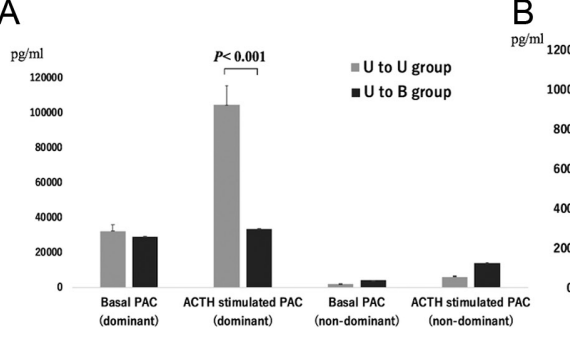

B

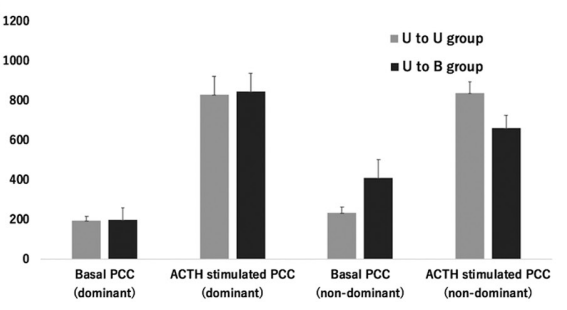

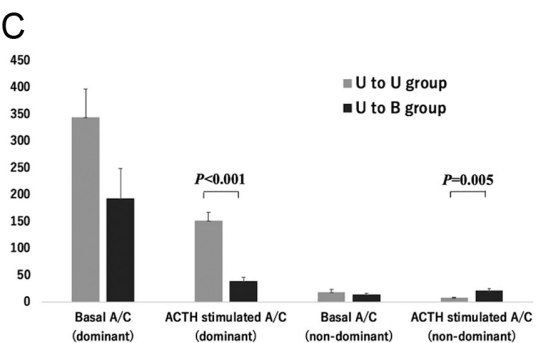

\section{Figure 4}

Changes in PAC (A), PCC (B), and A/C (C) ratio by ACTH stimulation on the dominant and non-dominant sides among patients with better surgical outcome in the $U$ to $B$ group and patients in the $U$ to $U$ group.

study that used different cut-off value series demonstrated that the number of patients diagnosed with bilateral hyperaldosteronism increased after ACTH stimulation with each cut-off value, which was corroborated by our results. In our study, the $U$ to $B$ group had the highest proportion of patients, implying that it is crucial in the clinical practice of PA to consider the surgical indication especially among patients of the $\mathrm{U}$ to $\mathrm{B}$ group.

As opposed to our results, El Ghorayeb et al. demonstrated that all eight patients in their study who showed lateralization using basal but not ACTHstimulated AVS data achieved clinical and biochemical cure/improvement and concluded that basal data was important (24). This result was based on a small sample size without the use of the PASO criteria (a wellrecognized international consensus for surgical outcome evaluation). In contrast, using a large sample size and the PASO criteria, we demonstrated for the first time that PA patients in the U to B group had worse clinical and biochemical outcomes and a lower rate of adrenal adenoma as a pathological finding compared with those in the $\mathrm{U}$ to $\mathrm{U}$ group. It is noteworthy that patients in the $\mathrm{U}$ to B group who underwent adrenalectomy had a higher detection rate of adrenal nodule on CT scan and higher pre- and post-ACTH stimulated LI than did those who did not undergo adrenalectomy. This therefore suggests that, if all patients in the $U$ to $B$ group were to undergo surgery, the surgical outcome would be far worse than that among patients in the $\mathrm{U}$ to $\mathrm{B}$ group who underwent adrenalectomy in our study. On the other hand, we could not find any significant difference in the surgical outcomes between the B to $\mathrm{U}$ and $\mathrm{U}$ to $\mathrm{U}$ groups. These results point to the important fact that ACTH-stimulated LI might be superior to basal LI in terms of the surgical outcomes.

We need to mention the discrepancy between the results obtained in this study and our previous report.
In our previous study, we demonstrated that ACTH stimulation during AVS did not affect the clinical and biochemical outcomes (18). This discrepancy may be due to the fact that the sample size of the present study was $27 \%$ higher than that of the previous study, thereby enhancing the statistical power of the present study.

Then, should we consider only ACTH-stimulated LI as an indication for surgery? To answer this question, our other findings are worth discussing. Although patients in the $\mathrm{U}$ to $\mathrm{B}$ group had worse surgical outcomes than did those in the $U$ to $U$ group, $43 \%$ of patients in the $\mathrm{U}$ to $\mathrm{B}$ group had both clinical and biochemical benefits after adrenalectomy and could be differentiated from those with worse surgical outcomes using basal LI and not ACTH-stimulated LI. Furthermore, the majority of patients with better surgical outcomes had no apparent recurrence of hyperaldosteronism during the long-term follow-up except one patient for whom adrenalectomy could have been avoided, given the high basal aldosterone levels obtained in both adrenal veins during AVS. These results imply that surgically curable APA and non-curable hyperaldosteronism, mainly composed of idiopathic hyperaldosteronism, were present in the $\mathrm{U}$ to $\mathrm{B}$ group

Interestingly, there was no difference in basal PAC on the dominant side in both the $U$ to $U$ and $U$ to B groups with better surgical outcomes. In contrast, there was a significant difference in the response of aldosterone to ACTH stimulation between the groups. Regarding these results, Rossi et al. discussed important issues in a recent review (12). The authors analyzed the entire transcriptome of APA in patients who were not preselected based on ACTH-stimulated AVS and compared the mRNA level of APA and the normal adrenal cortex of patients adrenalectomized due to renal cancer. This analysis revealed that the cosyntropin receptor MC2R is under-expressed in about two-thirds of patients with APA compared with patients with normal adrenal cortex (25). 
These differences in MC2R receptor expression explain the weak response in aldosterone level in a proportion of surgically curable APA cases. These results demonstrated that we might overlook some cases of surgically curable APA if only ACTH-stimulated LI was used.

Recently, the AVIS-2 Study group, which is the largest international study group, reported important results that the use of ACTH during AVS resulted in decreased lateralization while increasing the rate of studies judged to be successful (26). This implies that ACTH stimulation during AVS might obscure surgically curable cases of APA, and the authors recommended using unstimulated AVS as the optimal protocol. In the present study, we expanded upon the AVIS-2 results by showing that the use of data only from unstimulated AVS might lead to worse surgical outcomes than the use of ACTH-stimulated AVS results. Conversely, we also demonstrated that some patients with surgically curable APA could be differentiated using non-ACTH-stimulated LI in the U to B group. This result corroborates and clarifies the implications of AVIS-2 study. This study has several limitations. First, to make the discussion clear, we assessed only patients with bilaterally selective catheterization in both basal and ACTH-stimulated AVS with a strict cut-off value of LI. In this regard, it is well known that unselective sampling is more frequent in AVS without ACTH stimulation, which needs to be taken into account when applied in the clinical setting. Secondly, our results may have been affected by the retrospective study design, by the small subgroup size, and by the differences in clinical practice at each institution. Thirdly, the potential selection bias introduced by including only patients who underwent surgery must be considered. To decide on the clinically applicable LI for basal or ACTH-stimulated AVS, we need to include patients who did not undergo surgery, most of whom would have idiopathic hyperaldosteronism, to the analysis. Finally, we could not assess patients in the B to U group with sufficient statistical power due to the small number of patients in the group. As shown in Fig. 2, both clinical and biochemical benefits were lower in the B to $\mathrm{U}$ group than in the $\mathrm{U}$ to $\mathrm{U}$ group, although the $P$ value did not reach statistical significance. Furthermore, in the sensitivity analysis, the rate of adrenal adenoma as a pathological finding was significantly lower in the B to $\mathrm{U}$ group than in the $\mathrm{U}$ to $\mathrm{U}$ group $(P<0.001)$ (Supplementary Fig. 2). These findings indicate that, similar to patients in the $\mathrm{U}$ to $\mathrm{B}$ group, it is possible that patients in the $\mathrm{B}$ to $U$ group have a lower rate of surgical success than did those in the $U$ to $U$ group. Thus, this group should be investigated further.
This study showed that, although PA patients in the $\mathrm{U}$ to B group had worse surgical outcome than did those in the $\mathrm{U}$ to $\mathrm{U}$ group, basal LI could discriminate among patients with better surgical outcome in the $U$ to $B$ group. Our results may encourage clinicians to consider that using only ACTH-stimulated data during adrenal venous sampling might obscure some cases of surgically curable APA that can be differentiated using non-ACTHstimulated data.

\section{Supplementary materials}

This is linked to the online version of the paper at https://doi.org/10.1530/ EJE-19-0860.

\section{Declaration of interest}

The authors declare that there is no conflict of interest that could be perceived as prejudicing the impartiality of this study.

\section{Funding}

This research was supported by AMED, Japan (Grant Number JP17ek0109122 and JP19ek0109352) and the National Center for Global Health and Medicine, Japan (27-1402, 30-1008).

\section{Acknowledgements}

The acknowledgement list of JPAS and JRAS groups is available in the supplementary data of this article.

\section{References}

1 Funder JW, Carey RM, Mantero F, Murad MH, Reincke M, Shibata H, Stowasser M \& Young Jr WF. The management of primary aldosteronism: case detection, diagnosis, and treatment: an Endocrine Society clinical practice guideline. Journal of Clinical Endocrinology and Metabolism 2016101 1889-1916. (https://doi. org/10.1210/jc.2015-4061)

2 Mulatero P, Stowasser M, Loh KC, Fardella CE, Gordon RD, Mosso L, Gomez-Sanchez CE, Veglio F \& Young Jr WF. Increased diagnosis of primary aldosteronism, including surgically correctable forms, in centers from five continents. Journal of Clinical Endocrinology and Metabolism 200489 1045-1050. (https://doi.org/10.1210/jc.2003031337)

3 Rossi GP, Bernini G, Caliumi C, Desideri G, Fabris B, Ferri C, Ganzaroli C, Giacchetti G, Letizia C, Maccario M et al. A prospective study of the prevalence of primary aldosteronism in 1,125 hypertensive patients. Journal of the American College of Cardiology 200648 2293-2300. (https://doi.org/10.1016/j.jacc.2006.07.059)

4 Monticone S, Burrello J, Tizzani D, Bertello C, Viola A, Buffolo F, Gabetti L, Mengozzi G, Williams TA, Rabbia F et al. Prevalence and clinical manifestations of primary aldosteronism encountered in primary care practice. Journal of the American College of Cardiology 201769 1811-1820. (https://doi.org/10.1016/j.jacc.2017.01.052)

5 Catena C, Colussi G, Nadalini E, Chiuch A, Baroselli S, Lapenna R $\&$ Sechi LA. Cardiovascular outcomes in patients with primary aldosteronism after treatment. Archives of Internal Medicine 2008168 80-85. (https://doi.org/10.1001/archinternmed.2007.33)

6 Milliez P, Girerd X, Plouin PF, Blacher J, Safar ME \& Mourad JJ. Evidence for an increased rate of cardiovascular events in patients 
with primary aldosteronism. Journal of the American College of Cardiology 200545 1243-1248. (https://doi.org/10.1016/j. jacc.2005.01.015)

7 Rossi GP, Bernini G, Desideri G, Fabris B, Ferri C, Giacchetti G, Letizia C, Maccario M, Mannelli M, Matterello MJ et al. Renal damage in primary aldosteronism: results of the PAPY Study. Hypertension 200648 232-238. (https://doi.org/10.1161/01. HYP.0000230444.01215.6a)

8 Nishikawa T, Omura M, Satoh F, Shibata H, Takahashi K, Tamura N, Tanabe A \& Task Force Committee on Primary Aldosteronism, The Japan Endocrine Society. Guidelines for the diagnosis and treatment of primary aldosteronism - the Japan Endocrine Society 2009. Endocrine Journal 201158 711-721. (https://doi.org/10.1507/endocrj. EJ11-0133)

9 Rossi GP, Barisa M, Allolio B, Auchus RJ, Amar L, Cohen D, Degenhart C, Deinum J, Fischer E, Gordon R et al. The Adrenal Vein Sampling International Study (AVIS) for identifying the major subtypes of primary aldosteronism. Journal of Clinical Endocrinology and Metabolism 201297 1606-1614. (https://doi.org/10.1210/ jc.2011-2830)

10 Rossi GP, Pitter G, Bernante P, Motta R, Feltrin G \& Miotto D. Adrenal vein sampling for primary aldosteronism: the assessment of selectivity and lateralization of aldosterone excess baseline and after adrenocorticotropic hormone (ACTH) stimulation. Journal of Hypertension 200826 989-997. (https://doi.org/10.1097/ HJH.0b013e3282f9e66a)

11 Mulatero P, Bertello C, Sukor N, Gordon R, Rossato D, Daunt N, Leggett D, Mengozzi G, Veglio F \& Stowasser M. Impact of different diagnostic criteria during adrenal vein sampling on reproducibility of subtype diagnosis in patients with primary aldosteronism. Hypertension 201055 667-673. (https://doi.org/10.1161/ HYPERTENSIONAHA.109.146613)

12 Deinum J, Groenewoud H, Wilt GJV, Rossi G \& Lenzini L. Adrenal venous sampling: cosyntropin stimulation or not? European Journal of Endocrinology 2019181 D15-D26. (https://doi.org/10.1530/EJE-180844)

13 Satoh F, Abe T, Tanemoto M, Nakamura M, Abe M, Uruno A, Morimoto R, Sato A, Takase K, Ishidoya S et al. Localization of aldosterone-producing adrenocortical adenomas: significance of adrenal venous sampling. Hypertension Research 200730 1083-1095. (https://doi.org/10.1291/hypres.30.1083)

14 Monticone S, Satoh F, Giacchetti G, Viola A, Morimoto R, Kudo M, Iwakura Y, Ono Y, Turchi F, Paci E et al. Effect of adrenocorticotropic hormone stimulation during adrenal vein sampling in primary aldosteronism. Hypertension 201259 840-846. (https://doi. org/10.1161/HYPERTENSIONAHA.111.189548)

15 Nawata H, Demura H, Suda T \& Takayanagi R. Adrenal preclinical Cushing's syndrome. In Annual Report of the Japanese Ministry of Health and Welfare's 'Disorder of Adrenal Hormones' Research Committee, Japan, 1995, pp 223-226.

16 Williams TA, Lenders JWM, Mulatero P, Burrello J, Rottenkolber M, Adolf C, Satoh F, Amar L, Quinkler M, Deinum J et al. Outcomes after adrenalectomy for unilateral primary aldosteronism: an international consensus on outcome measures and analysis of remission rates in an international cohort. Lancet: Diabetes and Endocrinology 20175 689-699. (https://doi.org/10.1016/S2213-8587(17)30135-3)

17 Umakoshi H, Tsuiki M, Yokomoto-Umakoshi M, Takeda Y, Takashi Y, Kurihara I, Itoh $\mathrm{H}$, Katabami T, Ichijo $\mathrm{T}$, Wada $\mathrm{N}$ et al. Correlation between lateralization index of adrenal venous sampling and standardized outcome in primary aldosteronism. Journal of the Endocrine Society 20182 893-902. (https://doi.org/10.1210/js.201800055)

18 Takeda Y, Umakoshi H, Takeda Y, Yoneda T, Kurihara I, Katabami T, Ichijo T, Wada N, Yoshimoto T, Ogawa Y et al. Impact of adrenocorticotropic hormone stimulation during adrenal venous sampling on outcomes of primary aldosteronism. Journal of Hypertension 201937 1077-1082. (https://doi.org/10.1097/ HJH.0000000000001964)

19 Webb R, Mathur A, Chang R, Baid S, Nilubol N, Libutti SK, Stratakis CA \& Kebebew E. What is the best criterion for the interpretation of adrenal vein sample results in patients with primary hyperaldosteronism? Annals of Surgical Oncology 201219 1881-1886. (https://doi.org/10.1245/s10434-011-2121-5)

20 Wolley MJ, Ahmed AH, Gordon RD \& Stowasser M. Does ACTH improve the diagnostic performance of adrenal vein sampling for subtyping primary aldosteronism? Clinical Endocrinology 201685 703-709. (https://doi.org/10.1111/cen.13110)

21 Mathur A, Kemp CD, Dutta U, Baid S, Ayala A, Chang RE, Steinberg SM, Papademetriou V, Lange E, Libutti SK et al. Consequences of adrenal venous sampling in primary hyperaldosteronism and predictors of unilateral adrenal disease. Journal of the American College of Surgeons 2010211 384-390. (https:// doi.org/10.1016/j.jamcollsurg.2010.05.006)

22 Elliott P \& Holmes DT. Adrenal vein sampling: substantial need for technical improvement at regional referral centres. Clinical Biochemistry 201346 1399-1404. (https://doi.org/10.1016/j. clinbiochem.2013.04.004)

23 Seccia TM, Miotto D, De Toni R, Pitter G, Mantero F, Pessina AC \& Rossi GP. Adrenocorticotropic hormone stimulation during adrenal vein sampling for identifying surgically curable subtypes of primary aldosteronism: comparison of 3 different protocols. Hypertension 200953 761-766. (https://doi.org/10.1161/ HYPERTENSIONAHA.108.128553)

24 El Ghorayeb N, Mazzuco TL, Bourdeau I, Mailhot JP, Zhu PS, Thérasse E \& Lacroix A. Basal and post-ACTH aldosterone and its ratios are useful during adrenal vein sampling in primary aldosteronism. Journal of Clinical Endocrinology and Metabolism 2016 101 1826-1835. (https://doi.org/10.1210/jc.2015-3915)

25 Rossitto G, Maiolino G, Lenzini L, Bisogni V, Seccia TM, Cesari M, Iacobone M \& Rossi GP. Subtyping of primary aldosteronism with adrenal vein sampling: hormone- and side-specific effects of cosyntropin and metoclopramide. Surgery 2018163 789-795. (https://doi.org/10.1016/j.surg.2017.09.032)

26 Rossitto G, Amar L, Azizi M, Riester A, Reincke M, Degenhart C, Widimsky J, Mitsuhide N, Deinum J, Schultzekool L et al. Subtyping of primary aldosteronism in the AVIS-2 study: assessment of selectivity and lateralization. Journal of Clinical Endocrinology and Metabolism 2019 Epub. (https://doi.org/10.1210/clinem/dgz017)

Received 23 October 2019

Revised version received 5 December 2019

Accepted 3 January 2020 